\title{
High resolution near-infrared imaging of submillimeter galaxies
}

\author{
Paula Aguirre ${ }^{1,2}$, Andrew J. Baker ${ }^{3}$, Felipe Menanteau ${ }^{3}$, Dieter Lutz ${ }^{4}$ \\ and Linda J. Tacconi ${ }^{4}$ \\ ${ }^{1}$ Pontificia Universidad Católica de Chile, Depto. de Astronomía, Santiago, Chile. \\ ${ }^{2}$ Depto. de Ciencias Físicas, Facultad de Ciencias Exactas, U. Andrés Bello, Santiago, Chile \\ ${ }^{3}$ Department of Physics and Astronomy, Rutgers, the State University of New Jersey. \\ ${ }^{4}$ Max-Planck-Institut für extraterrestrische Physik,d Garching, Germany.
}

\begin{abstract}
We have obtained high-resolution F110W $(\sim J)$ and F160W $(\sim H)$ band observations of ten submillimeter galaxies (SMGs) with the Hubble Space Telescope's NICMOS camera, in order to resolve their rest-frame optical morphologies, determine the existence of multiplecomponent, merger-like configurations, and estimate their stellar masses. The selected targets have redshifts in the range $2.2 \leqslant z \leqslant 2.81$ confirmed with millimeter or mid-IR spectroscopy, guaranteeing that the two bands sample the galaxies' rest-frame optical light with the Balmer break falling between them.
\end{abstract}

Keywords. galaxies: evolution, galaxies: high-redshift, galaxies: peculiar

Morphologies: To investigate the possible multi-component, merger-like nature of our targets we used the maximum deblending option in SExtractor to assess whether each system could be resolved into two or more stellar structures coalescing to form a massive SMG. Through our photometric analysis and with the support of previous dynamical evidence, we classify five of our targets as multi-component systems and obtain F110W/F160W magnitudes for individual components. We interpret these system restframe optical and/or molecular gas morphologies as signs of ongoing mergers.

The high resolution and long wavelength of our HST imaging has also allowed us to characterize our targets with greater confidence than possible until now. As an example, we show in Fig. 1 the F110W and F160W images for SMM J04431+0210; the exceptional spatial resolution of our data allows us to distinguish for the first time two separate components (A and $\mathrm{B}$ ), which match the positions of two $\mathrm{CO}(3-2)$ peaks detected in this $\mathrm{z}=2.51$ SMG by Tacconi et al. (2006).

Stellar population synthesis modeling: We have modeled the stellar populations of eight objects with F110W and F160W photometry (including archival HST optical data when available) using Hyperzmass (Pozzetti et al. 2007) and the stellar synthesis library from Bruzual \& Charlot (2003). For multiple systems, we analyzed sub-components separately.

For each component, there are various stellar population models that provide equally good fits to the observed photometry. Thus, we cannot discriminate among star formation histories, but we can constrain stellar masses. After correction for lensing, we obtain a mean $\log \left(M^{*} / M_{\odot}\right)=$ $10.88 \pm 0.24$ for our full sample. For multiple systems, mass ratios range from $\sim 3: 1$ to $\sim 60: 1$, indicating that both

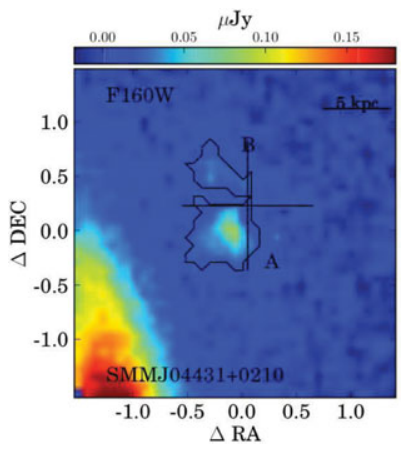

Figure 1. HST/NICMOS F160W imaging for the submillimeter galaxy SMM $\mathrm{J} 04431+0210$. major and minor mergers are represented in the SMG population. The morphologies 
and merger mass ratios of the least and most massive systems match the predictions of the major-merger and cold accretion (e.g., Davé et al. 2010) SMG formation scenarios, respectively, suggesting that both channels have a role in the population's origin.

\section{References}

Bruzual, G. \& Charlot, S. 2002, MNRAS 344, 1000

Davé, R., et al. 2010, MNRAS, 404, 1355

Ivison, R., et al. 2010, MNRAS, 404, 198

Pozzetti, L., et al. 2007 A $\mathscr{E} A, 474,443$

Swinbank, M., et al. 2005, MNRAS, 359, 401

Swinbank, M., et al. 2010, MNRAS, 405,234

Tacconi, L., et al. 2006, ApJ, 640, 228

Targett, T., et al. 2011, MNRAS, 412, 295 